Pacific

Journal of

Mathematics

LOWER BOUND ESTIMATES

OF THE FIRST EIGENVALUE FOR COMPACT MANIFOLDS

WITH POSITIVE RICCI CURVATURE

DAGANG YANG 


\title{
LOWER BOUND ESTIMATES \\ OF THE FIRST EIGENVALUE FOR COMPACT MANIFOLDS WITH POSITIVE RICCI CURVATURE
}

\author{
DAGANG YANG
}

\begin{abstract}
We present some new lower bound estimates of the first eigenvalue for compact manifolds with positive Ricci curvature in terms of the diameter and the lower Ricci curvature bound of the manifolds. For compact manifolds with boundary, it is assumed that, with respect to the outward normal, it is of nonnegative second fundamental form for the first Neumann eigenvalue and the mean curvature of the boundary is nonnegative for the first Dirichlet eigenvalue.
\end{abstract}

\section{Introduction.}

For a smooth $n$-dimensional closed Riemannian manifold $M^{n}$ whose Ricci curvature satisfies

$$
\operatorname{Ric}\left(M^{n}\right) \geq(n-1) K>0
$$

for some positive constant $K$, it has been shown by A. Lichnerowicz [6] in 1958 (see also [7]) that the first positive eigenvalue $\lambda$ of the manifold $M$ has a lower bound

$$
\lambda \geq n K
$$

The aim of this paper is to give some new lower bound estimates in terms of the lower Ricci curvature bound $(n-1) K$ and the diameter $d$ of the manifold $M$. The main results of this paper are summarized in the following two theorems.

Theorem 1. Let $M^{n}$ be a closed Riemannian manifold with $\operatorname{Ric}\left(M^{n}\right) \geq$ $(n-1) K \geq 0$ and diameter $d$. Then the first positive eigenvalue $\lambda$ on $M^{n}$ satisfies the lower bound

$$
\lambda \geq \frac{1}{4}(n-1) K+\frac{\pi^{2}}{d^{2}} .
$$

Theorem 2. Let $M^{n}$ be a compact manifold with nonempty boundary and with $\operatorname{Ric}\left(M^{n}\right) \geq(n-1) K \geq 0$. 
(a) Assume that the boundary $\partial M$ is weakly convex, that is, the second fundamental form with respect to the outward normal is nonnegative. Then the first positive Neumann eigenvalue $\lambda$ on $M^{n}$ satisfies the same lower bound (1.3).

(b) Assume that the mean curvature with respect to the outward normal of the boundary $\partial M$ is nonnegative. Then the first positive Dirichlet eigenvalue $\lambda$ on $M^{n}$ satisfies the lower bound estimate

$$
\lambda \geq \frac{1}{4}\left\{(n-1) K+\frac{\pi^{2}}{r^{2}}\right\}
$$

where $r$ is the inscribed radius for $M$.

These results generalize the Li-Yau [5] and Yang-Zhong [11] (cf. [4], [9]) estimates where they proved that the first positive eigenvalue satisfies $\lambda \geq$ $\frac{\pi^{2}}{d^{2}}$ for closed manifolds with nonnegative Ricci curvature. Notice that for manifolds with small diameter, Theorem 1 is better than the estimate (1.2) by A. Lichnerowicz. P. Li has conjectured that the first positive eigenvalue should satisfy the lower bound

$$
\lambda \geq(n-1) K+\frac{\pi^{2}}{d^{2}} .
$$

A proof of this conjecture would unify the Li-Yau and Yang-Zhong estimate for manifolds with nonnegative Ricci curvature with the Lichnerowicz estimate (1.1). It is my pleasure to thank Professor P. Li for raising to me this interesting problem.

Theorems 1 and 2 follow from Theorems 4.1 and 4.2, which are more precise statements of our results. Our ideas are based on the gradient estimate technique for eigenfunctions which was developed by P. Li and S.T. Yau $[\mathbf{3}],[\mathbf{5}]$. Some preliminary lemmas are proved in Section 2 and the gradient estimates of eigenfunctions are presented in Section 3. These estimates introduce a higher order term associated with the positive lower bound on the Ricci curvature in the gradient estimates of Li-Yau and Yang-Zhong. When $K=0$, our gradient estimates reduce to the estimates derived by Li-Yau and Yang-Zhong. The proof of Theorems 4.1 and 4.2 are presented in Section 4.

\section{Some Preliminary Lemmas.}

Throughout this paper, $M$ will be a compact $n$-dimensional Riemannian manifold with or without smooth boundary with $\operatorname{Ric}(M) \geq(n-1) K \geq 0$.

Let $v$ be a normalized eigenfunction of a positive eigenvalue $\lambda$ on $M$ with either Dirichlet or Neumann boundary condition if $\partial M \neq \emptyset$, that is,

$$
\Delta v=-\lambda v,
$$


such that

$$
\min v(x) \geq-1 \quad \text { and } \quad \max v(x)=1
$$

if $\partial M \neq \emptyset$ and $v$ is a Dirichlet eigenfunction, and

$$
\min v(x)=m_{1}-1 \quad \text { and } \quad \max v(x)=m_{1}+1
$$

if $\partial M \neq \emptyset$ and $v$ is a Neumann eigenfunction or $\partial M=\emptyset$, where $0 \leq m_{1}<1$ is the median of $v$.

To give a unified presentation of the three different cases, we set $m_{1}=0$ if $\partial M \neq \emptyset$ and $v$ is a Dirichlet eigenfunction. Let $u=v-m_{1}$. Given a constant $0<s<1$, consider the function

$$
w=s u=s v-m
$$

where $0 \leq m=s m_{1}<s<1$. Thus $\max |w(x)|=s<1$ and $\lim _{s \rightarrow 1^{-}} w=u$.

Lemma 2.1. Let $h(t)$ be a smooth positive function defined on the open interval $(-1,1)$. Assume that $\operatorname{Ric}(M) \geq(n-1) K$ and there is a point $p \in M$ such that the smooth function

$$
H=|\nabla w|^{2}-2 h(w)
$$

satisfies the conditions that

$$
H(p)=\max H(x)=0 \quad \text { and } \quad \nabla H(p)=0 .
$$

Then, at $t=w(p)$, the function $h$ satisfies the inequality

$$
h^{\prime 2}+\lambda(t+m) h^{\prime}+2 h\left\{(n-1) K-\lambda-h^{\prime \prime}\right\} \leq 0 .
$$

Proof. Let $t=w(p)$. Since $|w| \leq s<1, H(p)=0$, and $h$ is a positive smooth function on $(-1,1)$, we have

$$
|\nabla w(p)|^{2}=2 h(t)>0 .
$$

Choose a normal orthonormal frame $e_{1}, e_{2}, \ldots, e_{n}$ on a neighborhood of $p$ such that $e_{i} w(p)=0$ for $i>1$. For any smooth function $f$, we shall adopt the notation that $f_{i}=e_{i} f(p)$ and $f_{i j}=e_{j} e_{i} f(p)$ for $i, j=1,2, \ldots, n$. Then $w_{i}=0$ for $i>1$ and (2.8) implies that $w_{1}^{2}=2 h(t)>0$. Since $\nabla H(p)=0$, we have

$$
0=H_{j}=2 \sum_{i=1}^{n} w_{i} w_{i j}-2 h^{\prime}(t) w_{j}=2\left(w_{1} w_{1 j}-h^{\prime}(t) w_{j}\right)
$$

for $j=1,2, \ldots, n$. In particular,

$$
w_{11}=h^{\prime}(t) .
$$

Since $H$ attains its maximum at $p$ and $\nabla H(p)=0$, the maximum principle applies to give

$$
\Delta H(p) \leq 0 .
$$


It follows from Bochner's formula

$$
\frac{1}{2} \Delta|\nabla w|^{2}=\sum_{i, j=1}^{n} w_{i j}^{2}+\nabla w \cdot \nabla(\Delta w)+\operatorname{Ric}(\nabla w, \nabla w),
$$

$$
\begin{aligned}
0 & \geq \frac{1}{2} \Delta H(p) \\
& =\sum_{i j=1}^{n} w_{i j}^{2}-\lambda|\nabla w|^{2}+\operatorname{Ric}(\nabla w, \nabla w)-\left\{h^{\prime}(t) \Delta w+h^{\prime \prime}(t)|\nabla w|^{2}\right\} \\
& \geq h^{\prime 2}(t)+\lambda(t+m) h^{\prime}(t)+2 h(t)\left\{(n-1) K-\lambda-h^{\prime \prime}(t)\right\} .
\end{aligned}
$$

If the maximum value of $H$ is attained at an interior point $p$ in $M$, the condition that $\nabla H(p)=0$ in Lemma 2.1 is automatically satisfied. When $\partial M \neq \emptyset$ and $p \in \partial M$, the following lemma assures that $\nabla H(p)=0$ remains to be true if suitable convexity conditions are imposed on the boundary. Thus the maximum principle still applies even the maximum value of $H$ is attained on the boundary.

Lemma 2.2. Let $h(t)$ be a smooth positive function on the open interval $(-1,1)$, Assume that $\partial M \neq \emptyset$ and the maximum value 0 of the function (2.5) is attained at a boundary point $p \in \partial M$. Then $\nabla H(p)=0$ in either of the following two situations.

(a) $v$ is a Neumann eigenfunction and $\partial M$ is weakly convex in the sense that the second fundamental form $S$ in the outward normal direction is nonnegative definite.

(b) $v$ is a Dirichlet eigenfunction, $\partial M$ has nonnegative mean curvature $\operatorname{tr} S \geq 0$ in the outward normal direction, and $h(t)$ is an even function.

Proof. Let $p \in \partial M$ and $H(p)=\max H(x)=0$. We first consider the case where $v$ is a Neumann eigenfunction and assume that $\partial M$ is weakly convex. Let $e_{n}$ be the unit outward normal vector field on $\partial M$. Then $e_{n} w=0$ on $\partial M$ since $w=s v-m$ and $v$ is a Neumann eigenfunction. Let $e_{1}, e_{2}, \ldots, e_{n-1}$ be a local orthonormal frame tangent to $\partial M$ on a neighborhood of $p$ in $\partial M$ such that $w_{i}=e_{i} w(p)=0$ for $i>1$. Extend $e_{1}, e_{2}, \ldots, e_{n}$ to an orthonormal frame in a neighborhood of $p$ in $M$ by parallel translation along the geodesics $\exp _{\partial M}\left(t e_{n}\right)$. Thus $e_{n}=\frac{d}{d t} \exp _{\partial M}\left(t e_{n}\right)$ and $D_{e_{n}} e_{i}=0$ for $i=1,2, \cdots, n$, where $D$ is the covariant differential operator of the Riemannian manifold $M$. Moreover

$$
\nabla w(p)=w_{1} e_{1}(p) \neq 0
$$

since $|\nabla w(p)|^{2}=2 h(w(p))>0$ and $w_{i}=0$ for $i>1$. Since $\partial M$ is smooth, $e_{n} w(p)=0$, and $H$ has a maximum at $p \in \partial M$, we have $H_{i}=e_{i} H(p)=0$ 
for $i<n$ and

$$
\begin{aligned}
0 \leq H_{n} & =e_{n}|\nabla w(p)|^{2}=2 w_{1} e_{n} e_{1} w(p) \\
& =2 w_{1}\left\{e_{1} e_{n} w(p)+\left(D_{e_{n}} e_{1}\right) w(p)-\left(D_{e_{1}} e_{n}\right) w(p)\right\} \\
& =-2 w_{1}^{2}\left\langle D_{e_{1}} e_{n}, e_{1}\right\rangle
\end{aligned}
$$

where the last equality follows from the facts that $e_{n} w=0$ on $\partial M, e_{1}$ is tangent to $\partial M, D_{e_{n}} e_{1}=0$, and $w_{i}=0$ for $i>1$.

On the other hand, since $\partial M$ is weakly convex, that is, the second fundamental form $S$ satisfies $S(V, V)=\left\langle D_{V} e_{n}, V\right\rangle \geq 0$ for all tangent vector $V$ to $\partial M$, we obtain

$$
0 \leq H_{n}=-2 w_{1}^{2} S\left(e_{1}, e_{1}\right) \leq 0 .
$$

Hence $H_{n}=0$ and $\nabla H(p)=\sum_{i=1}^{n} H_{i} e_{i}(p)=0$.

Now let $h$ be an even function and let $v$ be a Dirichlet eigenfunction on $M$ with nonnegative mean curvature $\operatorname{tr} S \geq 0$. Extend $e_{n}$ to a local orthonormal frame $e_{1}, e_{2}, \ldots, e_{n}$ on a neighborhood of $p$ in $M$ such that $D_{e_{n}} e_{i}=0$ for $i=1,2, \ldots, n$. Recall that for Dirichlet boundary condition, we have $m_{1}=0$, thus $w=s v$ and $\left.w\right|_{\partial M}=s v_{\partial M}=0$. Therefore $\left.e_{i} w\right|_{\partial M}=0$ for $i<n$ and $\left.\nabla h(w)\right|_{\partial M}=0$ since $h$ is an even function. Since $H$ attains its maximum value at $p \in \partial M$, we have $H_{i}=0$ for $i<n$ and

$$
0 \leq H_{n}=e_{n}|\nabla w(p)|^{2}=2 \sum_{i=1}^{n} w_{i} e_{n} e_{i} w(p)=2 w_{n} e_{n}^{2} w(p) .
$$

Since $w(p)=0$ and $D_{e_{n}} e_{n}=0$, it follows from the definition of the Laplace operator that

$$
\begin{aligned}
0 & =-\lambda w(p)=\Delta w(p)=\sum_{i=1}^{n}\left(e_{i}^{2}-D_{e_{i}} e_{i}\right) w(p) \\
& =e_{n}^{2} w(p)+\bar{\Delta} w(p)-w_{n} \sum_{i=1}^{n-1}\left\langle e_{n}, D_{e_{i}} e_{i}\right\rangle
\end{aligned}
$$

where $\bar{\Delta}$ is the Laplace operator on $\partial M$ with the induced Riemannian metric. Since $\left.w\right|_{\partial M}=0$ and the mean curvature

$$
\operatorname{tr} S=\sum_{i=1}^{n-1}\left\langle D_{e_{i}} e_{n}, e_{i}\right\rangle=-\sum_{i=1}^{n-1}\left\langle e_{n}, D_{e_{i}} e_{i}\right\rangle
$$

is nonnegative, we obtain $\bar{\Delta} w(p)=0$ and

$$
0 \leq H_{n}=2 w_{n} e_{n}^{2} w(p)=-2 w_{n}^{2} \operatorname{tr} S(p) \leq 0 .
$$

Therefore $H_{n}=0$ and $\nabla H(p)=0$. 
We shall also need the following lower bound estimate of the first eigenvalue, which is due to $\mathrm{A}$. Lichnerowicz [6] when $M$ is a compact manifold without boundary. For completeness sake, a proof is enclosed.

Lemma 2.3. Assume that $\operatorname{Ric}(M) \geq(n-1) K>0$. Let $\lambda$ be the first positive eigenvalue on $M$ (with either Dirichlet or Neumann boundary condition if $\partial M \neq \emptyset)$. If $\partial M \neq \emptyset$, we also assume that $\partial M$ is of nonnegative mean curvature $\operatorname{tr} S \geq 0$ if $\lambda$ is a Dirichlet eigenvalue and $\partial M$ is of nonnegative definite second fundamental form $S \geq 0$ if $\lambda$ is a Neumann eigenvalue. Then

$$
\lambda \geq n K .
$$

Proof. Let $v$ be an eigenfunction of the eigenvalue $\lambda$. The lower bound (2.20) follows from integrating Bochner's formula for $\nabla v$

$$
\frac{1}{2} \Delta|\nabla v|^{2}=\sum_{i, j=1}^{n} v_{i j}^{2}-\lambda|\nabla v|^{2}+\operatorname{Ric}(\nabla v, \nabla v)
$$

on $M$ and applying the boundary conditions. More specifically, using the Schwarz inequality

$$
\sum_{i, j=1}^{n} v_{i j}^{2} \geq \sum_{i=1}^{n} v_{i i}^{2} \geq \frac{1}{n}(\Delta v)^{2}=\frac{1}{n} \lambda^{2} v^{2},
$$

and the lower bound on the Ricci curvature, integrate (2.21) over $M$ yields

$$
\frac{1}{2} \int_{\partial M} e_{n}|\nabla v|^{2}=\frac{1}{2} \int_{M} \Delta|\nabla v|^{2} \geq \int_{M}\left\{\frac{1}{n} \lambda^{2} v^{2}+[(n-1)-\lambda]|\nabla v|^{2}\right\} .
$$

Since $\Delta v=-\lambda v$, multiply by $v$ and integrate over $M$ and use the boundary conditions yield $\int_{M}|\nabla v|^{2}=\lambda \int_{M} v^{2}$. Hence

$$
\frac{1}{2} \int_{\partial M} e_{n}|\nabla v|^{2} \geq \frac{n-1}{n} \lambda(n K-\lambda) \int_{M} v^{2} .
$$

If $\partial M=\emptyset$, then (2.20) follows from (2.24) immediately. Otherwise, we show that $e_{n}|\nabla v|^{2} \leq 0$ pointwisely on $\partial M$ for either of the two boundary conditions. Indeed, for any $p \in \partial M$, choose an orthonormal frame $e_{1}, e_{2}, \ldots, e_{n}$ as in the proof of Lemma 2.2. For Neumann boundary condition, similar computations as in (2.14), (2.15), and the convexity condition $S \geq 0$ yield

$$
e_{n}|\nabla v|^{2}=-2 v_{1}^{2} S\left(e_{1}, e_{1}\right) \leq 0 .
$$

For Dirichlet boundary condition, similar computations as in (2.17), (2.18), (2.19), and $\operatorname{tr} S \geq 0$ yield

$$
e_{n}|\nabla v|^{2}=-2 v_{n}^{2} \operatorname{tr} S \leq 0 .
$$


In any case, we have

$$
\int_{\partial M} e_{n}|\nabla v|^{2} \leq 0
$$

Thus the lower bound estimate (2.20) follows from (2.24) and (2.27).

Notice that if we have a test function $h$ which satisfies the conditions in Lemma 2.1, then we get a gradient estimate

$$
|\nabla w|^{2} \leq 2 h(w)
$$

To construct a suitable test function $h$, the following function $z$, which was introduced by H.C. Yang and J.Q. Zhong [11] to estimate the first eigenvalue for manifolds with nonnegative Ricci curvature, is especially useful.

Lemma 2.4. The function

$$
z(t)=\frac{2}{\pi}\left(\arcsin t+t \sqrt{1-t^{2}}\right)-t
$$

is a continuous odd function on $[-1,1]$. Furthermore, on the open interval $(-1,1), z$ is smooth and satisfies

$$
\begin{gathered}
\left(1-t^{2}\right) z^{\prime \prime}+t z^{\prime}+t=0, \\
\frac{2}{5} t^{2}\left(1-t^{2}\right) \leq|z(t)|<\frac{1}{4}\left(1-t^{4}\right), \\
z^{\prime 2}-2 z z^{\prime \prime}>\frac{1}{4}\left(t-t z^{\prime}+2 z\right)^{2},
\end{gathered}
$$

$$
2\left(1-t^{2}\right)\left(3+t^{2}\right)\left(z^{\prime 2}-2 z z^{\prime \prime}+z^{\prime}\right)>\left\{6 t z+\left(1-t^{2}\right)\left(\frac{6}{\pi} \sqrt{1-t^{2}}-1\right)\right\}^{2} .
$$

Proof. It follows from the definition (2.29) for $z(t)$ that

$$
\begin{gathered}
z^{\prime}(t)=\frac{4}{\pi} \sqrt{1-t^{2}}-1, \\
z^{\prime \prime}(t)=-\frac{4}{\pi} t\left(1-t^{2}\right)^{-1 / 2} .
\end{gathered}
$$

Thus the identity (2.30) is clearly true. Furthermore, we have

$$
t-t z^{\prime}+2 z=\frac{4}{\pi} \arcsin t
$$

$$
z^{\prime 2}-2 z z^{\prime \prime}=\left(1-t^{2}\right)^{-1 / 2}\left\{\left(1-t^{2}\right)^{1 / 2}\left(1+\frac{16}{\pi^{2}}\right)-\frac{8}{\pi}+\frac{16}{\pi^{2}} t \arcsin t\right\},
$$


$(2.38)$

$z^{\prime 2}-2 z z^{\prime \prime}+z^{\prime}=4\left(1-t^{2}\right)^{-1 / 2}\left\{\frac{4}{\pi^{2}}\left[\left(1-t^{2}\right)^{1 / 2}+t \arcsin t\right]-\frac{1}{\pi}\left(1+t^{2}\right)\right\}$.

For the inequalities, we first notice that $z$ is an odd function. Hence, all of the functions involved in the inequalities are even functions. Therefore, we need only to verify them on the interval $[0,1)$.

Let

$$
\begin{gathered}
\phi(t)=z(t)-\frac{2}{5} t^{2}\left(1-t^{2}\right), \\
\phi_{1}(t)=1-t^{4}-4 z(t),
\end{gathered}
$$

$\phi_{2}(t)=\left(1-t^{2}\right)^{1 / 2}\left(1+\frac{16}{\pi^{2}}\right)-\frac{8}{\pi}+\frac{16}{\pi^{2}} t \arcsin t-\frac{4}{\pi^{2}}\left(1-t^{2}\right)^{1 / 2}(\arcsin t)^{2}$,

$\phi_{3}(t)=8\left(1-t^{2}\right)^{1 / 2}\left(3+t^{2}\right)\left\{\frac{4}{\pi^{2}}\left[\left(1-t^{2}\right)^{1 / 2}+t \arcsin t\right]-\frac{1}{\pi}\left(1+t^{2}\right)\right\}$

$$
-\left\{\frac{12}{\pi} t \arcsin t+\frac{6}{\pi}\left(1-t^{2}\right)^{1 / 2}\left(1+t^{2}\right)-1-5 t^{2}\right\}^{2} .
$$

Then the inequalities (2.31), (2.32), and (2.33) are equivalent to $\phi \geq 0$ and $\phi_{i}>0$ for $i=1,2,3$ on $[0,1)$. Since all of the functions are explicit elementary functions, it is easy to give a rigorous proof of these inequalities. However, it will take a few pages to do so. Instead, it is a much simpler matter to combine culculus with a graphing utility to verify these inequalities. The details will therefore be left to the readers.

\section{Gradient Estimates of Eigenfunctions.}

In this section, we prove the following gradient estimates.

Theorem 3.1. Let $M$ be a compact $n$-dimensional Riemannian manifold without boundary with $\operatorname{Ric}(M) \geq(n-1) K \geq 0$. Let $v$ be a normalized eigenfunction on $M$ with median $m_{1}$ of a positive eigenvalue $\lambda$. Let $u=$ $v-m_{1}, a=\frac{(n-1) K}{2 \lambda}$, and let $z$ be the function defined by (2.29). Then, the gradient of $u$ satisfies the inequality

$$
|\nabla u|^{2} \leq \lambda\left\{\left(1-u^{2}\right)\left[1-a\left(1-u^{2}\right)\right]+2 m_{1} z(u)\right\} .
$$

Theorem 3.2. Let $M$ be a compact n-dimensional Riemannian manifold with nonempty boundary and with $\operatorname{Ric}(M) \geq(n-1) K \geq 0$ and let $a=$ $\frac{(n-1) K}{2 \lambda}$. 
(a) Assume that the boundary is weakly convex, that is, the second fundamental form $S$ in the outward normal direction is nonnegative definite. Let $v$ be a normalized Neumann eigenfunction on $M$ with median $m_{1}$ of a positive eigenvalue $\lambda$. Then, the gradient of $u=v-m_{1}$ satisfies the same inequality (3.1).

(b) Assume that the boundary is of nonnegative mean curvature $\operatorname{tr} S \geq$ 0 in the outward normal direction. Let $v$ be a normalized Dirichlet eigenfunction on $M$ of a positive eigenvalue $\lambda$. Then the gradient of $v$ satisfies the inequality

$$
|\nabla v|^{2} \leq \lambda\left(1-v^{2}\right)\left\{1-a\left(1-v^{2}\right)\right\} .
$$

Notice that since $\lim _{s \rightarrow 1^{-}} m=m_{1}$ and $\lim _{s \rightarrow 1^{-}} w=v-m_{1}\left(m_{1}=0\right.$ for Dirichlet eigenfunction), to show the gradient estimates (3.1) and (3.2), it suffices to show the corresponding estimates for $w$. We shall use Lemma 2.1 twice. First, we show a gradient estimate for $w$ in Lemma 3.3 which is a slight variation of the Yang-Zhong [11] estimate for compact manifolds without boundary (see also $[\mathbf{4}]$ and $[\mathbf{9}]$ ).

Lemma 3.3. Assume that $\operatorname{Ric}(M) \geq 0$. If $\partial M \neq \emptyset$, we also assume that either the second fundamental form $S$ is nonnegative definite if $v$ is a Neumann eigenfunction or the mean curvature $\operatorname{tr} S$ is nonnegative if $v$ is a Dirichlet eigenfunction. Let $w=s v-m$ be as in Section 2. Then, for all $0<s<1$, the gradient of $w$ satisfies the inequality

$$
|\nabla w|^{2} \leq \lambda\left(1-w^{2}+2 m z(w)\right) .
$$

Proof. Since $|w| \leq s<1$ and $0 \leq m=s m_{1}<1$, the inequality (2.31) implies that $1-w^{2}+2 m z(w)$ is a positive smooth function on $M$. Thus, there exists a positive constant $\beta$ such that the smooth function

$$
Q=|\nabla w|^{2}-\beta\left(1-w^{2}+2 m z(w)\right)
$$

has 0 as its maximum value. Thus, the inequality (3.3) will follow if $\beta \leq \lambda$. Let

$$
h(t)=\frac{\beta}{2}\left(1-t^{2}+2 m z(t)\right) .
$$

Notice that if $v$ is a Dirichlet eigenfunction, then $m=s m_{1}=0$ and $h$ is an even function. Let $p \in M$ be a point where the function $Q$ attains its maximum value 0 . The convexity conditions $S \geq 0$ or $\operatorname{tr} S \geq 0$ and Lemma 2.2 implies that $\nabla Q(p)=0$. It follows from Lemma 2.1 that, at $t=w(p) \in(-1,1)$, the function $h$ defined by (3.5) satisfies the inequality (2.7) with $K=0$, namely,

$$
0 \geq h^{\prime 2}+\lambda(t+m) h^{\prime}-2 h\left(\lambda+h^{\prime \prime}\right) .
$$


Since $h^{\prime}=\beta\left(m z^{\prime}-t\right), h^{\prime \prime}=\beta\left(m z^{\prime \prime}-1\right)$, and $\beta>0$, divide the inequality (3.6) by $\beta$ and simplify yield

$$
\begin{aligned}
0 \geq & (\beta-\lambda)\left\{1+m\left(t+2 z-t z^{\prime}\right)+m^{2}\left(z^{\prime 2}-2 z z^{\prime \prime}\right)\right\} \\
& +\lambda m^{2}\left\{z^{\prime 2}-2 z z^{\prime \prime}+z^{\prime}\right\}-m \beta\left\{\left(1-t^{2}\right) z^{\prime \prime}+t z^{\prime}+t\right\} .
\end{aligned}
$$

The last term is 0 because of the identity (2.30). Completing the square in the first term yields

$$
\begin{aligned}
0 \geq & (\beta-\lambda)\left\{1+\frac{m}{2}\left(t+2 z-t z^{\prime}\right)\right\}^{2}+\lambda m^{2}\left\{z^{\prime 2}-2 z z^{\prime \prime}+z^{\prime}\right\} \\
& +(\beta-\lambda) m^{2}\left\{z^{\prime 2}-2 z z^{\prime \prime}-\frac{1}{4}\left(t+2 z-t z^{\prime}\right)^{2}\right\} .
\end{aligned}
$$

If $\beta>\lambda$, then, it follows from Lemma 2.4 that all of the three terms on the right side of the inequality (3.8) is nonnegative. Moreover, the first term is positive if $m=0$ and the last two terms are both positive if $m \neq 0$. That is certainly not possible since the left side of the inequality (3.8) is 0 . Hence, we must have $\beta \leq \lambda$.

The rest of this section will be devoted to the proof of Theorem 3.1 and 3.2. If $K=0$, Theorem 3.1 and 3.2 follows from Lemma 3.3. So assume that $\operatorname{Ric}(M) \geq K>0$. As already been noticed, we need only show that there exists a constant $\alpha \geq a=\frac{(n-1) K}{2 \lambda}$ such that $w$ satisfies the inequality

$$
|\nabla w|^{2} \leq \lambda\left\{\left(1-w^{2}\right)\left[1-\alpha\left(1-w^{2}\right)\right]+2 m z(w)\right\} .
$$

It follows from Lemma 3.3 that there exists a nonnegative constant $\alpha$ such that the function

$$
G=|\nabla w|^{2}-\lambda\left\{\left(1-w^{2}\right)\left[1-\alpha\left(1-w^{2}\right)\right]+2 m z(w)\right\}
$$

has 0 as its maximum value since $G$ is a strictly increasing linear function in $\alpha$ and

$$
G=|\nabla w|^{2}-\lambda\left(1-w^{2}+2 m z(w)\right)+\lambda \alpha\left(1-w^{2}\right)^{2} \leq \lambda \alpha\left(1-s^{2}\right)^{2}<0
$$

if $\alpha<0$.

Suppose, on the contrary, that $\alpha<a$. By Lemma 2.3, we have $\lambda \geq n K$. Thus

$$
0 \leq \alpha<a=\frac{(n-1) K}{2 \lambda} \leq \frac{n-1}{2 n}<\frac{1}{2} .
$$

It follows from the inequality (2.31) and (3.12) that the new test function

$$
h(t)=\frac{\lambda}{2}\left\{\left(1-t^{2}\right)\left[1-\alpha\left(1-t^{2}\right)\right]+2 m z(t)\right\}
$$

is a positive smooth function on $(-1,1)$. 
Let $p \in M$ be a point where the smooth function $G$ attains its maximum value 0 . As in the proof of Lemma 3.3, the convexity condition $S \geq 0$ or $\operatorname{tr} S \geq 0$ and Lemma 2.2 implies that $\nabla G(p)=0$. It follows from Lemma 2.1 that, at $t=w(p) \in(-1,1)$, the function defined by (3.13) satisfies the inequality

$$
h^{\prime 2}+\lambda(t+m) h^{\prime}+2 h\left\{(n-1) K-\lambda-h^{\prime \prime}\right\} \leq 0 .
$$

Since

$$
\begin{aligned}
h^{\prime}(t) & =\lambda\left\{t\left[2 \alpha\left(1-t^{2}\right)-1\right]+m z^{\prime}(t)\right\}, \\
h^{\prime \prime}(t) & =\lambda\left\{2 \alpha-1-6 \alpha t^{2}+m z^{\prime \prime}(t)\right\},
\end{aligned}
$$

and $(n-1) K=2 a \lambda$, divide the inequality (3.14) by $\lambda^{2}$ and then simplify it using the identity (2.30) yield

$$
\begin{aligned}
0 \geq & 2(a-\alpha)\left\{\left(1-t^{2}\right)\left[1-\alpha\left(1-t^{2}\right)\right]+2 m z\right\} \\
(3.17) \quad+2 \alpha t^{2}\left(1-t^{2}\right)\left[2-\alpha\left(1-t^{2}\right)\right] & \\
& +2 m \alpha t\left\{6 t z+\left(1-t^{2}\right)\left[\frac{6}{\pi} \sqrt{1-t^{2}}-1\right]\right\}+m^{2}\left(z^{\prime 2}-2 z z^{\prime \prime}+z^{\prime}\right) .
\end{aligned}
$$

It follows from the inequalities (2.31), (2.33), and (3.12) that the first term on the right side of the inequality (3.17) is positive while the second and the fourth terms are nonnegative, thus $m>0$ and $\alpha>0$. Furthermore, it follows from $1>2 a>2 \alpha>0,(2.31)$, and (3.17) that

$$
\begin{aligned}
0>\alpha & \left\{t^{2}\left(1-t^{2}\right)\left(3+t^{2}\right)+2 m t\left[6 t z+\left(1-t^{2}\right)\left(\frac{6}{\pi} \sqrt{1-t^{2}}-1\right)\right]\right. \\
& \left.+2 m^{2}\left(z^{\prime 2}-2 z z^{\prime \prime}+z^{\prime}\right)\right\} \\
\geq \alpha & \left(1-t^{2}\right)^{-1}\left(3+t^{2}\right)^{-1}\left\{t\left(1-t^{2}\right)\left(3+t^{2}\right)\right. \\
& \left.+m\left[6 t z+\left(1-t^{2}\right)\left(\frac{6}{\pi} \sqrt{1-t^{2}}-1\right)\right]\right\}^{2} \\
+ & m^{2} \alpha\left\{2\left(z^{\prime 2}-2 z z^{\prime \prime}+z^{\prime}\right)\right. \\
& \left.-\left(1-t^{2}\right)^{-1}\left(3+t^{2}\right)^{-1}\left[6 t z+\left(1-t^{2}\right)\left(\frac{6}{\pi} \sqrt{1-t^{2}}-1\right)\right]^{2}\right\} .
\end{aligned}
$$

Since $m>0$ and $\alpha>0$, the inequality (3.18) apparently contradicts with the inequality (2.33) in Lemma 2.4. Therefore, we have proved that there exists a constant $\alpha \geq a$ such that $w$ satisfies the gradient estimate (3.9) for each constant $0<s<1$. Taking the limit to the inequality (3.9) by letting $s \rightarrow 1^{-}$now yields the inequalities (3.1) and (3.2). This completes the proof of Theorems 3.1 and 3.2. 


\section{Lower Bound Estimates of the First Positive Eigenvalue.}

In this section, we apply the gradient estimates obtained in the previous section to derive some new lower bound estimates of the first positive eigenvalue on compact Riemannian manifolds whose Ricci curvature satisfies $\operatorname{Ric}(M) \geq(n-1) K \geq 0$.

Theorem 4.1. Let $M$ be a compact $n$-dimensional Riemannian manifold without boundary whose Ricci curvature satisfies $\operatorname{Ric}(M) \geq(n-1) K \geq 0$. Let $d$ be the diameter of $M$. Let $v$ be the normalized eigenfunction of the first positive eigenvalue $\lambda$ so that

$$
\inf v(x)=m_{1}-1 \quad \text { and } \quad \max v(x)=m_{1}+1
$$

where $0 \leq m_{1}<1$ is the median of $v$. Then

$$
\begin{aligned}
\lambda \geq & \min \left\{(n-1) K+\frac{\pi^{2}}{d^{2}},(n-1) K / 4+\frac{\pi^{2}}{d^{2}}\left[1+0.09 m_{1}^{2}\right]^{2}[1\right. \\
& \left.\left.+\sum_{k=1}^{\infty} \frac{(4 k-1) ! !(2 k-1) ! !}{(4 k) ! !(2 k) ! !}\left(\frac{(n-1) K d^{2}}{4 \pi^{2}+3(n-1) K d^{2}}\right)^{2 k}\right]^{2}\right\} .
\end{aligned}
$$

Proof. Let $u=v-m_{1}$. It follows from Theorem 3.1 that

$$
|\nabla u|^{2} \leq \lambda\left\{\left(1-u^{2}\right)\left[1-a\left(1-u^{2}\right)\right]+2 m_{1} z(u)\right\}
$$

where $a=\frac{(n-1) K}{2 \lambda}$. Hence

$$
\begin{array}{r}
|\nabla u| \\
\left(1-u^{2}\right)^{1 / 2}\left[1-a\left(1-u^{2}\right)\right]^{1 / 2}\left[1+2 m_{1} z(u)\left(1-u^{2}\right)^{-1}\left[1-a\left(1-u^{2}\right)\right]^{-1}\right]^{1 / 2} \\
\leq \lambda^{1 / 2}
\end{array}
$$

By (4.1), there exist two points $p, q \in M$ such that

$$
u(p)=-1 \quad \text { and } \quad u(q)=1 .
$$

Let $\gamma(t)$ be a minimal geodesic from $p$ to $q$ in $M$ and let

$$
\theta(x)=\arcsin u(x) \in[-\pi / 2, \pi / 2] .
$$


Integrate (4.4) along $\gamma$ yields

$$
\begin{aligned}
\lambda^{1 / 2} d \geq & \int_{\gamma} \lambda^{1 / 2} d t \geq \int_{-1}^{1}\left(1-u^{2}\right)^{-1 / 2}\left[1-a\left(1-u^{2}\right)\right]^{-1 / 2}\{1 \\
& \left.+2 m_{1} z(u)\left(1-u^{2}\right)^{-1}\left[1-a\left(1-u^{2}\right)\right]^{-1}\right\}^{-1 / 2} d u \\
= & \int_{-\pi / 2}^{\pi / 2}\left[1-\frac{a}{2}-\frac{a}{2} \cos 2 \theta\right]^{-1 / 2}\left\{1+2 m_{1} z(\sin \theta) \sec ^{2} \theta[1\right. \\
& \left.\left.-\frac{a}{2}-\frac{a}{2} \cos 2 \theta\right]^{-1}\right\}^{-1 / 2} d \theta .
\end{aligned}
$$

Let $b=\frac{a}{2-a}=\frac{(n-1) K}{4 \lambda-(n-1) K}$. Then $0 \leq b<1 / 3$ since $\lambda \geq n K$. Thus

$$
\left[1-\frac{a}{2}-\frac{a}{2} \cos 2 \theta\right]^{-1 / 2}=(1-a / 2)^{-1 / 2}(1-b \cos 2 \theta)^{-1 / 2} \geq 1 .
$$

The inequality (2.31) in Lemma 2.4 implies that

$$
2 m_{1}|z(\sin \theta)| \sec ^{2} \theta\left[1-\frac{a}{2}-\frac{a}{2} \cos 2 \theta\right]^{-1} \leq m_{1}<1
$$

since $a<1 / 2$.

So we can apply the binomial series expansion

$$
(1-y)^{-1 / 2}=1+\sum_{k=1}^{\infty} \frac{(2 k-1) ! !}{(2 k) ! !} y^{k}
$$

for

$$
y=-2 m_{1} z(\sin \theta) \sec ^{2} \theta\left[1-\frac{a}{2}-\frac{a}{2} \cos 2 \theta\right]^{-1}
$$

and notice that (4.11) is an odd function in $\theta$. It follows from (4.7), (4.8), (4.10), and (4.11) that

$$
\begin{aligned}
\lambda^{1 / 2} d \geq & (1-a / 2)^{-1 / 2} \int_{-\pi / 2}^{\pi / 2}[1-b \cos 2 \theta]^{-1 / 2}[1 \\
& \left.+\sum_{k=1}^{\infty} \frac{(2 k-1) ! !}{(2 k) ! !} y^{k}\right] d \theta \\
= & 2(1-a / 2)^{-1 / 2} \int_{0}^{\pi / 2}[1-b \cos 2 \theta]^{-1 / 2}[1 \\
& \left.+\sum_{k=1}^{\infty} \frac{(4 k-1) ! !}{(4 k) ! !} y^{2 k}\right] d \theta \\
\geq & 2(1-a / 2)^{-1 / 2} \int_{0}^{\pi / 2}[1-b \cos 2 \theta]^{-1 / 2}\left[1+\frac{3}{8} y^{2}\right] d \theta .
\end{aligned}
$$


By the inequalities (2.31) and (4.8), we have

$$
y^{2} \geq\left[\frac{4}{5} m_{1} \sin ^{2} \theta\right]^{2}=\frac{16}{25} m_{1}^{2} \sin ^{4} \theta .
$$

Now expand $(1-b \cos 2 \theta)^{-1 / 2}$ in (4.12) and integrate term by term yield

$$
\begin{aligned}
& d \lambda^{1 / 2}\left(1-\frac{a}{2}\right)^{1 / 2} \\
& \geq 2 \int_{0}^{\pi / 2}\left(1+\frac{6}{25} m_{1}^{2} \sin ^{4} \theta\right)(1-b \cos 2 \theta)^{-1 / 2} d \theta \\
& =2 \int_{0}^{\pi / 2}\left(1+\frac{6}{25} m_{1}^{2} \sin ^{4} \theta\right)\left[1+\sum_{k=1}^{\infty} \frac{(2 k-1) ! !}{(2 k) ! !} b^{k} \cos ^{k} 2 \theta\right] d \theta \\
& \geq \pi\left(1+0.09 m_{1}^{2}\right)\left[1+\sum_{k=1}^{\infty} \frac{(4 k-1) ! !(2 k-1) ! !}{(4 k) ! !(2 k) ! !} b^{2 k}\right] .
\end{aligned}
$$

Since $a=\frac{(n-1) K}{2 \lambda}$, we obtain

$$
\lambda \geq \frac{n-1}{4} K+\frac{\pi^{2}}{d^{2}}\left(1+0.09 m_{1}^{2}\right)^{2}\left[1+\sum_{k=1}^{\infty} \frac{(4 k-1) ! !(2 k-1) ! !}{(4 k) ! !(2 k) ! !} b^{2 k}\right]^{2}
$$

where $b=\frac{(n-1) K}{4 \lambda-(n-1) K}$.

So either $\lambda \geq \frac{\pi^{2}}{d^{2}}+(n-1) K$ or else $b \geq \frac{(n-1) K d^{2}}{4 \pi^{2}+3(n-1) K d^{2}}$ and

$$
\begin{aligned}
\lambda \geq & \frac{n-1}{4} K+\frac{\pi^{2}}{d^{2}}\left(1+0.09 m_{1}^{2}\right)^{2} \\
& \cdot\left[1+\sum_{k=1}^{\infty} \frac{(4 k-1) ! !(2 k-1) ! !}{(4 k) ! !(2 k) ! !}\left(\frac{(n-1) K d^{2}}{4 \pi^{2}+3(n-1) K d^{2}}\right)^{2 k}\right]^{2} .
\end{aligned}
$$

This completes the proof of Theorem 4.1.

Theorem 4.2. Let $M$ be a compact $n$-dimensional Riemannian manifold with nonempty boundary $\partial M$. Assume that the Ricci curvature satisfies $\operatorname{Ric}(M) \geq(n-1) K \geq 0$. Let $d=\operatorname{diam}(M)$ be the diameter of $M$ and let $r=\sup \{d(x, \partial M) \mid x \in M\}$ be the inscribed radius of $M$. Then:

(a) If the second fundamental form of the boundary in the outward normal direction is nonnegative definite, then the first positive Neumann eigenvalue for $M$ satisfies the same inequality (4.2).

(b) If the mean curvature of the boundary in the outward normal direction is nonnegative, then the first positive Dirichlet eigenvalue for $M$ 
satisfies the lower bound

$$
\begin{aligned}
\lambda \geq & \min \left\{(n-1) K+\frac{\pi^{2}}{4 r^{2}}, \frac{n-1}{4} K\right. \\
& \left.+\frac{\pi^{2}}{4 r^{2}}\left[1+\sum_{k=1}^{\infty} \frac{(4 k-1) ! !(2 k-1) ! !}{(4 k) ! !(2 k) ! !}\left(\frac{(n-1) K r^{2}}{\pi^{2}+3(n-1) K r^{2}}\right)^{2 k}\right]^{2}\right\} .
\end{aligned}
$$

Proof. Since the Neumann eigenfunction satisfies the same gradient estimate (3.1), the proof of the lower bound (4.2) for the first positive Neumann eigenvalue is identical with the proof of Theorem 4.1. The proof of the lower bound (4.17) for the first Dirichlet eigenvalue is also similar to the proof of Theorem 4.1.

Let $v$ be the normalized first Dirichlet eigenfunction such that $0 \leq v \leq$ $\max v(x)=1$. Let $q \in M$ and $p \in \partial M$ be two points such that $v(q)=1$ and $d(p, q)=d(q, \partial M)$. By the definition of the inscribed radius $r$, we have $d(p, q) \leq r$. It follows from the inequality (3.2) that

$$
\frac{|\nabla v|}{\left(1-v^{2}\right)^{1 / 2}\left[1-a\left(1-v^{2}\right)\right]^{1 / 2}} \leq \lambda^{1 / 2} .
$$

Integrate the inequality (4.18) along a minimal geodesic from $p$ to $q$ as in the proof of Theorem 4.1 yields the desired lower bound (4.17).

\section{References}

[1] J. Cheeger, A lower bound for the smallest eigenvalue of the Laplacian, Problems in Analysis, a symposium in honor of S. Bochner, Princeton Univ. Press, Princeton, 1970.

[2] J. Escobar, Uniqueness theorems on conformal deformation of metrics, Sobolev inequalities, and an eigenvalue estimate.

[3] P. Li, A lower bound for the first eigenvalue of the Laplacian on a compact Riemannian manifold, Indiana Univ. Math. J., 28 (1979), 1013-1019.

[4] L L Lecture Notes on Geometric Analysis, Lecture Notes Series, \#6, Seoul National University, Seoul, Korea.

[5] P. Li and S.T. Yau, Eigenvalues of a compact Riemannian manifold, AMS Proc. Symp. Pure Math., 36 (1980), 205-239.

[6] A. Lichnerowicz, Géometrie des groupes de transformations, Dunod, Paris, 1958.

[7] M. Obata, Certain conditions for a Riemannian manifold to be isometric to the sphere, J. Math. Soc. Japan, 14 (1962), 333-340.

[8] R. Reilly, Applications of the Hessian operator in a Riemannian manifold, Indiana Univ. Math. J., 26 (1977), 459-472.

[9] R. Schoen and S.T. Yau, Lectures on Differential Geometry, Conference Proceedings and Lecture Notes in Geometry and Topology, Vol. I, International Press, 1994. 
[10] S.T. Yau, Isoperimetric constants and the first eigenvalue of a compact Riemannian manifold, Ann. Scient. Éc. Norm. Sup., 4 (1985), 487-507.

[11] H.C. Yang and J.Q. Zhong, On the estimate of the first eigenvalue of a compact Riemannian manifold, Sci. Sinica Ser., A27 (1984), 1265-1273.

Received September 30, 1997. This research was partially supported by NSF grant 9404312.

Tulane University

New ORLEANS, LA 70118

E-mail address: dgy@math.tulane.edu 